EPJ Web of Conferences 66, 02008 (2014)

DOI: $10.1051 /$ epjconf/ 20146602008

(C) Owned by the authors, published by EDP Sciences, 2014

\title{
Investigation of $0^{+}$states in mercury isotopes after two-neutron transfer
}

\author{
C. Bernards ${ }^{1}$,a , R.F. Casten ${ }^{1}$, V. Werner ${ }^{1}$, P. von Brentano², D. Bucurescu ${ }^{3}$, G. Graw ${ }^{4}$, S. Heinze ${ }^{2}$, \\ R. Hertenberger ${ }^{4}$, J. Jolie ${ }^{2}$, S. Lalkovski ${ }^{5}$, D.A. Meyer ${ }^{1}$, D. Mücher ${ }^{2, b}$, P. Pejovic ${ }^{1}$, C. Scholl ${ }^{2}$, and \\ H.-F. Wirth ${ }^{6}$
}

${ }^{1}$ Wright Nuclear Structure Laboratory, Yale University, New Haven, CT-06520, USA

${ }^{2}$ Institut für Kernphysik, Universität zu Köln, D-50937 Köln, Germany

${ }^{3}$ National Institute for Physics and Nuclear Engineering, Bucharest, R-76900, Romania

${ }^{4}$ Fakultät für Physik, Ludwig-Maximilians-Universität München, D-85748 Garching, Germany

${ }^{5}$ Faculty of Physics, University of Sofia, 1164 Sofia, Bulgaria

${ }^{6}$ Physik Department, Technische Universität München, D-85748 Garching, Germany

\begin{abstract}
Using the high-resolution Q3D magnetic spectrograph at the Maier-Leibnitz Laboratory (MLL) Tandem accelerator in Munich, we studied $0^{+}$excitations in the mercury isotopes ${ }^{198} \mathrm{Hg},{ }^{200} \mathrm{Hg}$, and ${ }^{202} \mathrm{Hg}$ after two-neutron transfer. We only observed 4-6 excited $0^{+}$states per nucleus up to about $3-\mathrm{MeV}$ excitation energy, far fewer than in other experiments of this $(p, t)$ campaign. The results reveal a sharp drop in the number of low-lying $0^{+}$states towards the ${ }^{208} \mathrm{~Pb}$ shell closure. We discuss the low-energy $0^{+}$state density as a function of the valence nucleon number $N_{\text {val }}$. The $0^{+}$excitation energies and the measured $(p, t)$ transfer cross sections indicate a structural change throughout the $\mathrm{Hg}$ isotopes, with the most notable result being the peaking in the cross section of the low-lying excited $0_{2}^{+}$state in ${ }^{200} \mathrm{Hg}$.
\end{abstract}

\section{Introduction}

Because of its capability to measure the characteristic forward-peaking of $L=0$ transfers, the Q3D magnetic spectrograph [1] at the Maier-Leibnitz Laboratory (MLL) Tandem accelerator in Munich -in combination with the focal plane detector [2]- has turned out to be a very successful instrument for the identification of $0^{+}$excitations. This feature has been extensively used to identify $0^{+}$states throughout the rare-earth region from gadolinium up to platinum [3-7]. For some nuclei, the number of identified $0^{+}$states increased sharply with the analysis of the Q3D measurement. The large number of low-lying $0^{+}$states in ${ }^{154} \mathrm{Gd}[8]$ was interpreted as a new signature for the shape-phase transition from spherical to deformed nuclei [9]. The unexpected high number of observed $0^{+}$excitations triggered various calculations reproducing the density and distribution of $0^{+}$states [10-13]. Now, with the experiments on the even mercury isotopes ${ }^{198-202} \mathrm{Hg}[14,15]$, we move further to the ${ }^{208} \mathrm{~Pb}$ shell closure. This allows us to investigate $0^{+}$states in the vicinity of the prolate-oblate shape-phase transition in the

\footnotetext{
a e-mail: christian.bernards@yale.edu

${ }^{b}$ Present address: Physik Department, Technische Universität München, D-85748 Garching, Germany
}

This is an Open Access article distributed under the terms of the Creative Commons Attribution License 2.0, which permits unrestricted use, distribution, and reproduction in any medium, provided the original work is properly cited. 
Table 1. Assigned $0^{+}$states in ${ }^{198,200,}{ }^{202} \mathrm{Hg}$ and their relative $R(5 / 17.5)$ ratio. Newly assigned $0^{+}$states are marked with an asterisks, tentative $\left(0^{+}\right)$assignments are denoted in italic. Each $\mathrm{Hg}$ isotope is labeled with its corresponding valence neutron number $N_{\text {val }}$.

\begin{tabular}{llllll}
\hline \multicolumn{2}{c}{${ }^{198} \mathrm{Hg}\left(N_{\text {val }}=10\right)$} & \multicolumn{2}{c}{${ }^{200} \mathrm{Hg}\left(N_{\text {val }}=8\right)$} & \multicolumn{2}{c}{${ }^{202} \mathrm{Hg}\left(N_{\text {val }}=6\right)$} \\
Energy $(\mathrm{keV})$ & $\mathrm{R}(5 / 17.5)$ & Energy $(\mathrm{keV})$ & $\mathrm{R}(5 / 17.5)$ & Energy $(\mathrm{keV})$ & $\mathrm{R}(5 / 17.5)$ \\
\hline $0.0(0)$ & $13.3(4)$ & $0.0(0)$ & $13.4(4)$ & $0.1(1)$ & $15.7(4)$ \\
$1401.0(3)$ & $9.4(15)$ & $1029.3(1)$ & $4.8(2)$ & $1411.0(3)$ & $12.3(18)$ \\
$1646.4(8)^{*}$ & $>3.35$ & $1515.5(3)$ & $10.5(12)$ & $1643.0(3)$ & $7.3(5)$ \\
$1779.6(2)$ & $4.9(6)$ & $1856.6(2)$ & $8.0(21)$ & $1655.8(13)$ & $3.1(13)$ \\
& & $2246.1(2)^{*}$ & $5.0(7)$ & $1778.9(6)$ & $4.5(15)$ \\
& & $2331.8(3)$ & $3.2(9)$ & $2126.7(7)$ & $3.2(17)$ \\
& $2475.2(1)^{*}$ & $4.4(5)$ & $2570.7(10)$ & $2.3(25)$ \\
& & & & $2598.5(2) *$ & $4.3(4)$ \\
& & & & $2685.7(5)$ & $4.7(32)$ \\
\hline
\end{tabular}

$\mathrm{Hf}-\mathrm{Hg}$ region [16] and to test if the prolate-oblate shape-phase transition affects the low-energy $0^{+}$ state density of these nuclei.

\section{Experiment \& Analysis}

To identify $L=0$ transfers from the $\mathrm{Hg} 0^{+}$ground state (GS) in the target material to an excited $0^{+}$ state of the $\mathrm{Hg}$ isotope of interest, e.g., a transfer from the ${ }^{200} \mathrm{Hg}$ GS to an excited $0^{+}$state in ${ }^{198} \mathrm{Hg}$, we measured spectra at $5^{\circ}, 17.5^{\circ}$, and $30^{\circ}$ laboratory angle relative to the incoming proton beam. A more detailed description of the experimental setup at the Q3D, the specifications of the enriched $\mathrm{Hg}$ targets, and the analysis is given in Refs. $[14,15]$. The characteristic forward-peaking of $L=0$ transfers was determined by evaluating the ratio $R(5 / 17.5) \equiv \sigma\left(5^{\circ}\right) / \sigma\left(17.5^{\circ}\right)$ for each observed transfer. As a safe lower limit to prevent incorrect $0^{+}$assignments, we used $R(5 / 17.5)>3$.

\section{Results}

The resulting $0^{+}$assignments for ${ }^{198} \mathrm{Hg},{ }^{200} \mathrm{Hg}$, and ${ }^{202} \mathrm{Hg}$-based on the $R(5 / 17.5)$ ratio of our dataare listed in Table 1. In total, we assigned four new $0^{+}$states up to $\sim 3-\mathrm{MeV}$ excitation energy in these three $\mathrm{Hg}$ isotopes investigated. Tentative assignments are mostly due to a poor population of these particular states. Absolute cross sections for each state are given in Refs. $[14,15]$. Some $0^{+}$ assignments in the data sheets [17-19] were not confirmed. Whenever possible, we tested assignments from the $(p, t)$ data for consistency with $\gamma \gamma$ coincidence data of a fusion-evaporation experiment [20] and data of a neutron-capture experiment at ILL Grenoble [21, 22].

\section{Discussion}

Figure 1 shows the complete data set of $0^{+}$excitations assigned in this $(p, t)$ campaign at the Q3D spectrograph, ranging from $\mathrm{Gd}$ to $\mathrm{Hg}$ isotopes. One notes a significantly higher $0^{+}$state density in ${ }^{154} \mathrm{Gd}$, which was interpreted as a new signature for the shape-phase transition from spherical to deformed nuclei [8]. The prolate-oblate shape-phase transition observed in Ref. [16] does not show an effect on the low-energy $0^{+}$density. The number of low-lying $0^{+}$states rather declines with larger nucleon mass, down to only four low-energy $0^{+}$assignments in ${ }^{198} \mathrm{Hg}$ and ${ }^{202} \mathrm{Hg}$. 


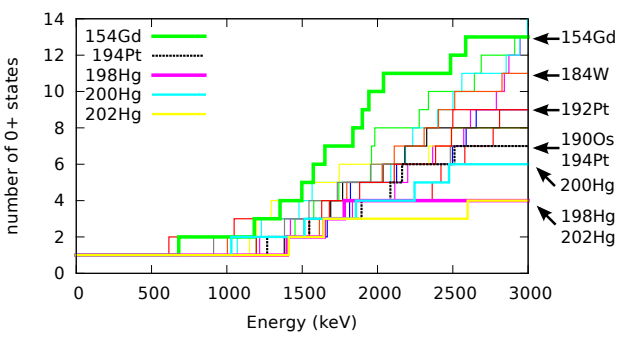

Figure 1. Histogram of low-energy $0^{+}$states observed in this $(p, t) \mathrm{Q} 3 \mathrm{D}$ campaign. Data are taken from Refs. $[3-7,14,15]$. The labeled nuclei on the right-hand side show the decline of $0^{+}$state density throughout the prolate-oblate shape-phase transitional region, with ${ }^{194} \mathrm{Pt}$ concluded to be the closest to the critical point [16]. Figure based on Ref. [14].

The effect of a decline in the number of low-energy $0^{+}$states towards the ${ }^{208} \mathrm{~Pb}$ shell closure is reproduced by Interacting Boson Model (IBM) [23] calculations and illustrated in Fig. 2. Please see Ref. [14] for more details on the calculations. Figure 2 shows that the number of observed $0^{+}$states strongly increases with $N_{\text {val }}$ as the valence space expands, but saturates near midshell starting at about $N_{\text {val }}=22$ valence nucleons.

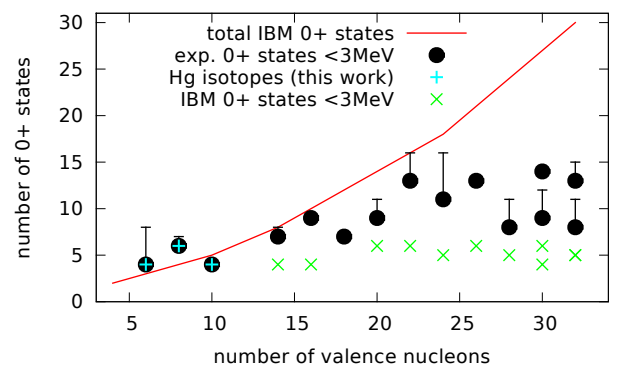

Figure 2. Number of $0^{+}$states up to $3 \mathrm{MeV}$ assigned in this $(p, t)$ campaign as a function of valence nucleon number $N_{\text {val }}$. The error bars include tentative assignments. The red line corresponds to the maximum number of $s d$ IBM $0^{+}$states, where as the green crosses indicate the calculated number of $0^{+}$states using realistic parameters, if available. Based on Ref. [15].

Figure 2 shows a small peak at $N_{\text {val }}=8$, corresponding to ${ }^{200} \mathrm{Hg}$. By directly comparing further properties of the investigated $\mathrm{Hg}$ isotopes one notes more differences shown in Fig. 3: the sequence and level energies of the low-lying states change rapidly at ${ }^{200} \mathrm{Hg}$ and the $0_{2}^{+}$state in ${ }^{200} \mathrm{Hg}$ is strongly populated with about $12 \%$ of the ground-state cross section. Historically, $0^{+}$state two-nucleon transfer cross sections approaching or exceeding $15 \%$ have signaled structural effects such as phase transitional regions or shape coexistence [9]. In Ref. [24], the enhanced $0_{2}^{+}$cross section in ${ }^{200} \mathrm{Hg}$ has been associated with an oblate single particle energy gap, but other explanations like mixing or coexistence cannot be ruled out. Another indicator for structural changes, the two-neutron separation energy $\mathrm{S}_{2 n}$ (or the differential $\delta \mathrm{S}_{2 n}$ ), shows at most a weak anomaly $[25,26]$.

\section{Conclusion}

The experiments on the $\mathrm{Hg}$ isotopes complete a high-resolution $(p, t)$ campaign using the Q3D spectrograph. We observe fewer low-energy $0^{+}$states in ${ }^{198,200,}{ }^{202} \mathrm{Hg}$ than in other nuclei investigated in this $(p, t)$ campaign. Plotted as a function of valence nucleon number $N_{\text {val }}$, we note a sharp drop in the number of $0^{+}$states in the near-magic region compared to the transitional and deformed regions, but a saturation towards midshell. The low-energy $0^{+}$density seems not to be affected by the prolate-oblate shape phase transition [16] in the Hf-Hg region.

The $0_{2}^{+}$state in ${ }^{200} \mathrm{Hg}$ has a large two-nucleon transfer cross section, which might be an indicator for a structural change throughout the $\mathrm{Hg}$ isotopes at $N=120$. To understand this effect, it would be very helpful to learn more about the $E 0$ ground-state transition of the $0_{2}^{+}$state.

We acknowledge support by the US DOE under Grant No. DE-FG02-91ER-40609. 

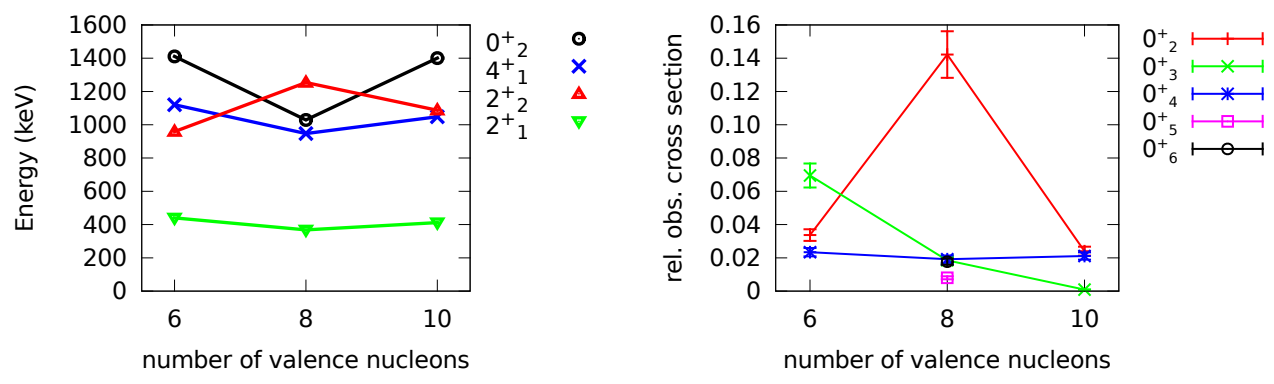

Figure 3. Low-energy states (left) and relative observed cross sections of $0^{+}$excitations (right) for ${ }^{198,} 200,202 \mathrm{Hg}$ as a function of valence nucleon number $N_{\text {val }}$. The significant changes in excitation energies for the low-lying states and the unusual strong population of the $0_{2}^{+}$state at $N_{\text {val }}=8$, corresponding to ${ }^{200} \mathrm{Hg}$, are often interpreted as an indicator for a structural change. Based on Ref. [15].

\section{References}

[1] M. Löffler, H.J. Scheerer, and H. Vonach, Nucl. Instr. Methods 111, 1 (1973).

[2] H.-F. Wirth, Ph.D. thesis, Technische Universität München, 2001.

[3] S.R. Lesher et al., Phys. Rev. C 66, 051305(R) (2002).

[4] D.A. Meyer et al., Phys. Rev. C 74, 044309 (2006).

[5] D. Bucurescu et al., Phys. Rev. C 73, 064309 (2006).

[6] L. Bettermann et al., Phys. Rev. C 80, 044333 (2009).

[7] G. Ilie et al., Phys. Rev. C 82, 024303 (2010).

[8] D.A. Meyer et al., Phys. Lett. B638, 44 (2006).

[9] P. Cejnar, J. Jolie, and R.F. Casten, Rev. Mod. Phys. 82, 2155 (2010).

[10] N.V. Zamfir, Jing-ye Zhang, and R.F. Casten, Phys. Rev. C 66, 057303 (2002).

[11] Yang Sun et al., Phys. Rev. C 68, 061301(R) (2003).

[12] N. Lo Iudice, A.V. Sushkov, and N.Yu. Shirikova, Phys. Rev. C 70, 064316 (2004).

[13] N. Lo Iudice, A.V. Sushkov, and N.Yu. Shirikova, Phys. Rev. C 72, 034303 (2005).

[14] C. Bernards et al., Phys. Rev. C 87, 024318 (2013).

[15] C. Bernards et al., Phys. Rev. C 87, 064321 (2013).

[16] J. Jolie and A. Linnemann, Phys. Rev. C 68, 031301(R) (2003).

[17] Z. Chunmei, Nuclear Data Sheets 95, 59 (2002).

[18] F.G. Kondev and S. Lalkovski, Nuclear Data Sheets 108, 1471 (2007).

[19] S. Zhu and F.G. Kondev, Nuclear Data Sheets 109, 699 (2008).

[20] C. Bernards et al., Phys. Rev. C 79, 054307 (2009).

[21] W. Urban et al., JINST 8, P03014 (2013).

[22] C. Bernards et al., Phys. Rev. C 84, 047304 (2011).

[23] F. Iachello and A. Arima, The Interacting Boson Model (Cambridge University Press, Cambridge, UK, 1987).

[24] M. Vergnes et al., Nucl. Phys. A514, 381 (1990).

[25] S. Anghel, G. Cata-Danil, and N.V. Zamfir, Rom. J. Phys. 54, 301 (2009).

[26] R.B. Cakirli, R.F. Casten, and K. Blaum, Phys. Rev. C 82, 061306(R) (2010). 